\title{
What is MRS All About, Really?
}

Most people experience the Materials Research Society (MRS) through the Fall and Spring Meetings, or through MRS publications such as the MRS Bulletin that you are reading now, the Journal of Materials Research, or Materials $360^{\circledR}$ online. But, as you can see from the 2008-2009 Annual Report elsewhere in this issue, MRS is much more. With the hope that you might find MRS services or activities that are of interest to you, here is a primer on MRS.

Probably the best way to get an overview of "what MRS is about" is to look at the MRS Constitution, Mission, and Strategic Objectives.

The MRS Constitution is the Society's legal founding document. It states our purpose at the highest level and changes only very rarely. According to this document, "The objective of this Society is to serve professionals working in the field of materials science and engineering by... fostering interaction among materials scientists and engineers... promoting an interdisciplinary approach... sponsoring interdisciplinary meetings... and disseminating information relevant to the materials community." It is important to recognize that MRS was not created to support any one industry, geographical region, group of people, or class of materials, but rather to promote technical excellence in the whole field of materials research using an interdisciplinary approach. In short, MRS was founded to be inclusive and egalitarian, a tradition that I am proud to say is alive and well today.

The MRS Mission follows from the Constitution, and quickly summarizes our charge: The Materials Research Society (MRS) is an organization of materials researchers from academia, industry, and government that promotes communication for the advancement of interdisciplinary materials research to improve the quality of life.

The MRS Strategic Objectives provide a written description of areas where the Society is striving to focus its efforts in the mid-term (5-10 years). The MRS Board of Directors reviewed and revised the MRS Strategic Objectives this year. The current MRS Strategic Objectives are:

Expand as the "Materials Gateway" for global communication and knowledge

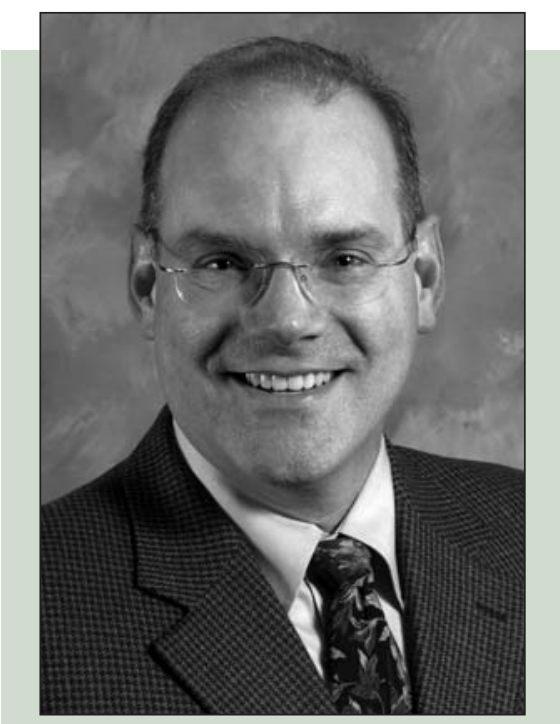

rials research and education to improve the quality of life by increasing the engagement of the scientific community, and catalyzing technological advancements in targeted areas.

Enhance the MRS community through expanding membership, diversity, and globalization. The Materials Research Society could provide services to a much larger fraction of the worldwide materials research community by identifying and reaching out to groups that are not well represented within MRS. Thus, MRS works to find ways to bring benefits and services to materials researchers, regardless of discipline, throughout the world.

Summing it up, MRS strives to provide the best technical information through meetings and publications, to support the community by encouraging policymakers and the public to fund materials research, to engage in developing technical solutions to challenging problems facing society, and to provide better services to a wider range of materials researchers.

But how, exactly, do we do these things? By a range of regular business activities as well as a steady stream of new initiatives. New initiatives arise from our volunteers. The MRS Board of

dissemination. The Materials Research Society provides and enables forums for the global exchange of technical information related to leading-edge, interdisciplinary materials research. Through its publications, Web site, and meetings, MRS seeks to serve the broadest possible community.

Support the sustainability of the field of materials research. Materials research provides real tangible benefits to society. However, like all of science, materials research thrives only if society supports it with funding and a steady flow of talented people. The Materials Research Society will effectively communicate the importance of science in society to the general public.

Proactively foster materials solutions to improve the quality of life. Materials research will provide the key elements for new technologies to address global needs. As such, the Materials Research Society promotes interdisciplinary mate-
Directors is facilitating broad-ranging initiatives in meetings and communications, but grassroots efforts-efforts proposed and implemented by our engaged and talented members - have been highly successful. Recent examples include the Strange Matter traveling museum exhibition, our increased advocacy initiatives, the new workshop series developed by the Women in Materials Science and Engineering group, the MRS Bulletin special issue on energy, the Science as Art competition, and many others.

So that is a snapshot of the guiding principles, goals, and objectives of MRS. If you have comments, particularly about what we are trying to accomplish with our strategic objectives (and if you have ideas for new initiatives), please send them to info@mrs.org, or to any MRS Board Member (see www.mrs.org/ board). It is, of course, your Society!

SHEFFORD P. BAKER 2009 MRS President 